\title{
The effect of rotational degree and routine activity on the risk of collapse in transtrochanteric rotational osteotomy for osteonecrosis of the femoral head-a finite element analysis
}

\author{
Pusheng Xie ${ }^{1,2,3} \cdot$ Yuping Deng ${ }^{1,2,3} \cdot$ Jinchuan Tan ${ }^{1,2,3} \cdot$ Mian Wang ${ }^{1,2,3} \cdot$ Yang Yang $^{1,2,3} \cdot$ Hanbin Ouyang ${ }^{4}$. \\ Wenhua Huang ${ }^{1,2,3,4}$ (D)
}

Received: 13 April 2019 / Accepted: 22 January 2020 / Published online: 3 February 2020

(C) The Author(s) 2020

\begin{abstract}
To explore the mechanical mechanism and provide preoperative planning basis for transtrochanteric rotational osteotomy (TRO) procedure, a joint-preserving procedure for osteonecrosis of the femoral head. Eleven TRO finite element femurs with the most common types of necrosis were analyzed under multi-loading conditions. Thereafter, we made a comprehensive evaluation by considering the anatomy characters, daily activities, and risk indicators contain necrosis expansion trend, necrotic blood supply pressure, and the risk of fracture. The risk of fracture (ROF) is the lowest when standing on feet and increases gradually during normal walking and walking upstairs and downstairs. Compared with posterior rotation, rotating forward keeps more elements at low risk. Additionally, the correlation analysis shows it has a strong negative correlation $\left(R^{2}=0.834\right)$ with the average modulus of the roof. TRO finally decreased the stress and energy effectively. However, the stress and strain energy arise when rotated posteriorly less than $120^{\circ}$. The comprehensive evaluation observed that rotating forward $90^{\circ}$ could reduce the total risks to $64 \%$. TRO is an effective technique to prevent collapse. For the anterior and superior large necrosis, we recommend to rotate forward $60^{\circ}$ to $90^{\circ}$ (more efficient) or backward $180^{\circ}$. The methodology followed in this study could provide accurate and personalize preoperative planning.
\end{abstract}

Keywords Osteonecrosis of the femoral head $\cdot$ Transtrochanteric rotational osteotomy $\cdot$ Routine activity $\cdot$ Risk of collapse $\cdot$ Finite element analysis

Pusheng Xie, Yuping Deng and Jinchuan Tan contributed equally to this work.

Hanbin Ouyang

Robin85@163.com

Wenhua Huang

Orthobiomech@163.com

Pusheng Xie

sepsen2014@163.com

Yuping Deng

b116ruce@126.com

Jinchuan Tan

453242077@qq.com

Mian Wang

476619444@qq.com

Yang Yang

15625055563@163.com
1 National Key Discipline of Human Anatomy, School of Basic Medical Sciences, Southern Medical University, 1023 ShaTai Rd, Guangzhou 510515, People's Republic of China

Department of Anatomy, School of Basic Medicine Science, Guangdong Provincial Key laboratory of Medical Biomechanics, Southern Medical University, 1023 ShaTai Rd, Baiyun District, Guangzhou 510515, People's Republic of China

Guangdong Engineering Research Center for Translation of Medical 3D Printing Application, Southern Medical University, 1023 ShaTai Rd, Guangzhou 510515, People's Republic of China

4 Orthopaedic Center, Affiliated Hospital of Guangdong Medical University, Guangdong Medical University, Zhanjiang 524002, People's Republic of China 


\section{Introduction}

Osteonecrosis of the femoral head (ONFH) remains an important concern because of the increasing morbidity and high disability rate. This disease appearing as a nearly silent may lead to progressive collapse followed by degenerative arthritis of the hip [1]. ONFH occurs mainly in younger and more active patients because potentially joint replacement procedure in these circumstances is associated with high rates of failure and there has been a continued search for procedures which preserve the hip [2-4].

Transtrochanteric rotational osteotomy (TRO) has been devised as a salvage surgical treatment because it can decompress the femoral head and improve the biomechanical properties in routine activity. It is an ideal procedure moving the remained viable femoral head area to the weight-bearing locale below the acetabular roof; thus, hip joints can be preserved for long term by reducing the stresses on the necrotic zone. If there is any intact area at the anterior or posterior portion of the femoral head, then the femoral head can be rotated to move this area to the load-bearing portion.

Rotational osteotomies such as anterior rotational osteotomy posterior rotational osteotomy have been proposed. And the effect of the operation is impressive [5-7]. However, some others were unable to reproduce his results and had to give up this technically demanding procedure. Reasons for the outcome variability include improper patient selection, anatomical variation in the blood supply of the femoral head, and especially, inadequacy in preoperative plan [8-10]. The importance of rotational direction and degree has been demonstrated by some researchers $[11,12]$. However, few scholars have addressed the problem of the mechanics mechanism after TRO, especially for the daily postoperative activities. In the present study, we applied finite element models with convergence analysis and mechanical experiment validation to analyze the necrosis expansion trend, necrotic blood supply pressure, and the risk of fracture by considering four routine activities after operation. The approach we have used aims to quantify the effect of rotational degree and load case on postoperative efficacy. And we tried to figure out if there were some correlation between ROF and modulus of bone. It provides theoretical basis for the selection of rotational direction and the best rotational angle.

\section{Materials and methods}

\subsection{Geometry extraction and finite element mesh generation}

A bone structure model of the proximal femur was reconstructed using Mimics 14.0 software (Materialise, Leuven, Belgium) from a series of computed tomography images of a 34-year-old male patient with large necrosis (grade C) [13] in anterior and superior position of the femoral head. This model was imported into the Unigraphics NX 8.5 software (Siemens, Munich, Germany) to simulate TRO surgery and then 11 groups of solid model were obtained (Fig. 1). The tetrahedron elements were used to generate mesh models by Abaqus 6.14 (Dassault Systemes, Velizy-Villacoublay, France). Finally, a total of 44 different mesh models simulating 4 different load cases with an intact femur and 10 different TRO femurs were created.

\subsection{Material properties}

As long as the heterogeneity of proximal femur was fully taken into account, the anisotropy of femur could be ignored. In this study, femur was modeled as an elastic and nonhomogenous material by assigning specific Young's modulus to each element by use of Mimics. In order to calculate each element's modulus of elasticity, the Hounsfield unit (HU) was extracted from $\mathrm{CT}$ images and was calculated at the centroid of each element. CT intensity values were utilized in assigning mechanical properties for interior cortical and trabecular bone, modeled as a continuum of bony tissue. An intensity value for each element was calculated by averaging the brightness of pixels within the element. The bone mineral density phantom established a linear relationship between the $\mathrm{CT}$ scanner pixel intensity (HU) and the calcium equivalent density $\rho$. An empirical relationship specific to bone was used to convert interior bone density to elastic modulus for each element. Equations 1 and 2 were then used to find the apparent density $\left(\rho, \mathrm{g} / \mathrm{cm}^{3}\right)$ and Young's modulus $(E, \mathrm{MPa})$ of each element, respectively [14]. And Poisson's ratio $v$ was set to be 0.3 .

$$
\begin{aligned}
& \rho=0.00069141 \times \mathrm{HU}+1.026716 \\
& E=2017.3 \rho^{2.46}
\end{aligned}
$$

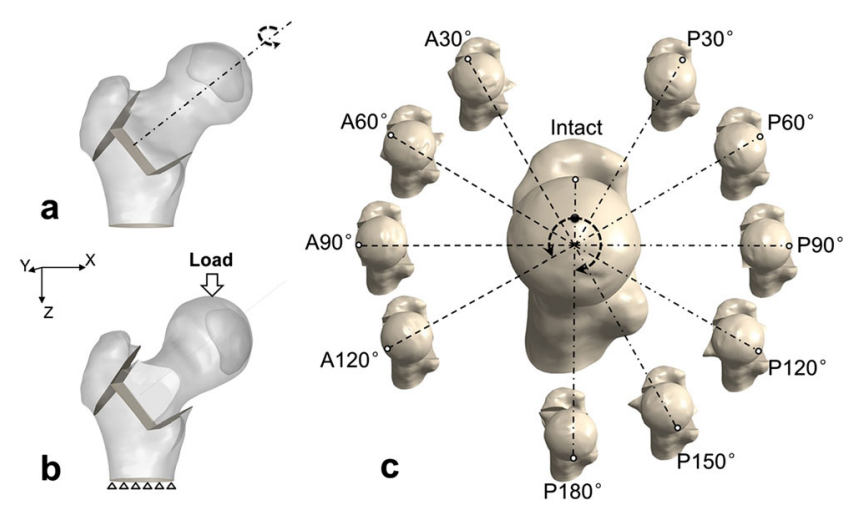

Fig. 1 Transtrochanteric rotational osteotomy operation schematic diagram. Osteotomy was performed via three resected surface, and then the femur head was rotated through the neck axis (a).The boundary and loading condition were applied on the TRO femur (b). Different rotational degrees (c) 


\subsection{Boundary and loading conditions}

The distal ends of the femoral models were fully constrained as boundary condition. Four loading cases simulated the peak loading of feet standing and normal walking upstairs and downstairs were applied on the femur as recorded by Bergmann et al. [15] (Table 1). The major weight-bearing zone of the acetabulum is defined by a $40^{\circ}$ angle around a vertical line passing through the center of the femoral head [16].

\subsection{Convergence test of the FEA models}

The convergences of the FEA models in this study were justified by the maximum von Mises stress of the intact proximal femur. Five different models were created to perform the convergence test. A mesh was generally considered to be sufficiently refined when an increase in mesh resolution yielded roughly a $5 \%$, or less, change in the result [17]. Based on the convergence test (Table 2), the average element size of $1.4 \mathrm{~mm}$ (percentage differences within 5\%) was chosen as the base model. And we validated the model by comparing the maximum principal stress and displacement from previous studies $[18,19]$.

\subsection{Mechanical experiment validation}

Literature verification and biomechanical experiment validation were performed in this study. Five fourth-generation composite femurs were examined with the same finite element modeling method mentioned above. And we carried out biomechanical experiments to test its axial stiffness and averaged strain value in the front area (left side of Fig. 6d) of the proximal femur with digital image correlation (DIC) method. And then the consistency analysis of axial stiffness and average strain of two groups were conducted to verify the effectiveness of our FEA method. The specific mechanical experiment verification method would be described below.

In order to reduce the error between samples and compare with related researches, we choose fourth-generation mediumsized composite bones (model number 3403) from Sawbones

Table 1 Joint contact forces for the four routine activities

\begin{tabular}{llccl}
\hline Load case & Magnitude(BW) & \multicolumn{4}{l}{ Unit vector } \\
\cline { 3 - 5 } & & $x$ & $y$ & $z$ \\
\hline Feet standing & 0.33 & 0 & 0 & 0.33 \\
Normal walking & 2.38 & -0.544 & -0.325 & 2.249 \\
Upstairs & 2.51 & -0.601 & -0.614 & 2.367 \\
Downstairs & 2.60 & -0.599 & -0.387 & 2.533 \\
\hline
\end{tabular}

The magnitude is in units of body weight (BW)

A body weight of $600 \mathrm{~N}$ was assumed for the present analyses

The axis $x$ is parallel to the transverse plane and pointed to the interior, $y$ is toward the front, and $z$ is parallel to the idealized midline of the femur and toward inferior
(Pacific Research Laboratories, Inc., Vashon Island, WA, USA) as the verification object. Each specimen was aligned under a simulated single leg stance (Fig. 6a). The force line (the line between the femoral head center and the femoral condyle center) was adjusted to the vertical direction by a 3D-printing fixture. The $5-\mathrm{cm}$ distal femur was embedded and fixed by polymethyl methacrylate (PMMA). Then the anterior surfaces of the composite bones were painted with spray-paint for DIC with white background color and a black random speckle pattern (Fig. 6c) as previously reported [20]. This dyeing method does not alter the mechanical properties of the samples [21].

The axial stiffness tests were conducted by an electronic universal testing machine (ATES6010, China). The specimens were first pre-loaded with the testing machine in position to control a vertical load of up to $100 \mathrm{~N}$. And then the loader was controlled to press down at a rate of $1 \mathrm{~mm} / \mathrm{min}$ until the force is up to $2100 \mathrm{~N}$. Loads and displacements were recorded and for at least $90 \mathrm{~s}$ after load removal for each repetition. Three loading repetitions were performed for each loading configuration, with the specimen being allowed to recover at least $10 \mathrm{~min}$ between replicates. Loads and displacements were collected by sensors. According to the load-displacement data, the slope of the linear region is calculated as the axial stiffness of the sample.

During mechanical testing, the anterior surfaces of the composite bones were recorded by a non-contact optical three-dimensional measuring system (ARAMIS 4M, GOM Corp., Germany) using DIC as previously reported [21, 22]. The two digital cameras with a frame rate of $4 \mathrm{~Hz}$ and pan angle of $22^{\circ}$ to medial from anterior view created a stereo view of the surface from which the 3D surface, displacements, and surface strains were calculated using the DIC program.

According to the aforementioned method, five corresponding sawbones finite element models were constructed. After the mesh convergence analysis was performed, the same boundary and loading conditions were used for axial stiffness analysis. The force, displacement, and average strain of the region of interest were calculated and compared with the results of biomechanical experiments.

\subsection{Data analysis}

The 11 groups of FEA model that applied 4 different loading conditions were investigated. For each case, the percentage change in the mechanical conditions of the treated femur in relation to the intact condition was evaluated in two different regions: (1) the roof of femoral head, (2) the necrotic area. A parameter was defined to quantify the risk of fracture (ROF) of the femoral head [23]:

$\mathrm{ROF}=\varepsilon_{\max } / \varepsilon_{\lim }$

where $\varepsilon_{\max }$ is the maximum absolute principal strain at each element of the model (either compressive or tensile) and $\varepsilon_{\lim }$ is 
Table 2 Convergence test of the FEA models

\begin{tabular}{llllll}
\hline & Case 1 & Case 2 & Case 3 & Case 4 & Case 5 \\
\hline Element type & C3D10 & & & & \\
Size (mm) & 1.2 & 1.6 & 2 & 2.4 & 1.4 \\
Element numbers & 497,741 & 238,810 & 140,499 & 94,302 & 358,645 \\
Node numbers & 706,316 & 342,582 & 202,997 & 137,130 & 510,962 \\
Max stress (MPa) in femur and changed ratio (\%) & 34.29 & 32.05 & 29.41 & 27.7 & 35.29 \\
& $(0)$ & $(6.53)$ & $(14.23)$ & $(19.22)$ & $(0.29)$ \\
Max stress (MPa) in necrosis and changed ratio (\%) & 11.42 & 12.08 & 9.972 & 11.41 & 11.44 \\
& $(0)$ & $(5.779)$ & $(12.68)$ & $(0.088)$ & $(0.175)$ \\
\hline
\end{tabular}

the corresponding ultimate strain (ultimate tensile strain $\varepsilon_{\lim }^{T}$ $=0.0073$ and ultimate compressive strain $\varepsilon_{\lim }^{C}=0.0104$ ) [24].

The maximum absolute principal strain was determined at each element for each of the intact femur. These elements were classified in four groups with the same number of elements by quartiles, this corresponds to I, II, III, and IV levels of ROF, respectively [25]. The higher level indicates higher risk of collapse. Then, the strain values of the TRO femurs were classified according to the groups/quartiles mentioned above. ROF greater than 1 means collapse happened. Thereafter, the number of elements falling into the different strain categories was quantified in the TRO femurs and compared with those of the intact femur. In addition, the average von Mises stress and all strain energy (ALLSE) were calculated to investigate the necrotic area.

\subsection{Statistical methods}

All the data were collected and analyzed by SPSS Statistics v20 software (IBM, Armonk, NY, USA). The presented box plots represent median and first and third quartiles. The error bars indicate maximum and minimum values. Descriptive statistics and Pearson's correlation analysis are adopted in the course of analysis.

\section{Results}

\subsection{ROF}

As shown in Fig. 2, the percentage of ROF of the roof area after TRO was presented in four levels. Comparing with posterior rotational osteotomy, anterior rotational osteotomy could keep more elements at a lower level of ROF. Among the four load conditions, the ROF is the lowest when standing on both feet and increases gradually during normal walking and walking upstairs and downstairs. The average and the extremal ROF graphs that reach the upper and lower bounds were given (Fig. 3). In general, the ROFs of TRO models under four load cases were all increased but less than 1 and remained at a low level after TRO. We hypothesize that it may be caused by the different property of the anterior or posterior portion of the femoral head. In order to make sure if that is related to the modulus of the roof area, we sorted out the average modulus of the roof area from all TRO models under feet standing load case. As presented in Fig. 4, it shows a strong negative correlation $\left(R^{2}=0.834\right)$. In other words, the better the bone quality at the roof of the femoral head, the lower the risk of collapse.

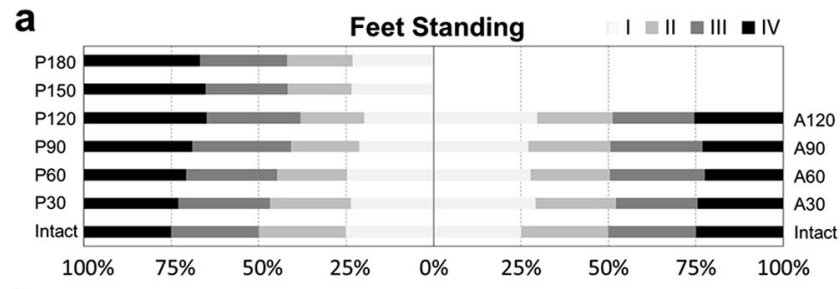

b
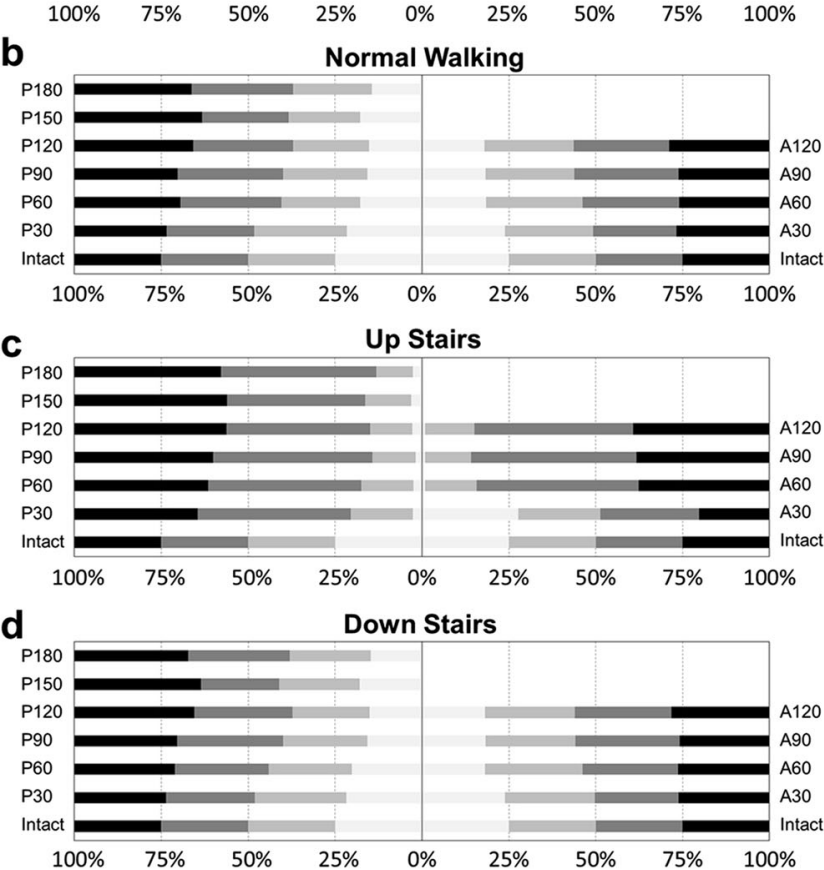

Fig. 2 Comparison of the four levels ROF after different TRO under four load cases in relation to the intact femur 
Fig. 3 Boxplots about the ROF of the femurs after TRO under four different load cases
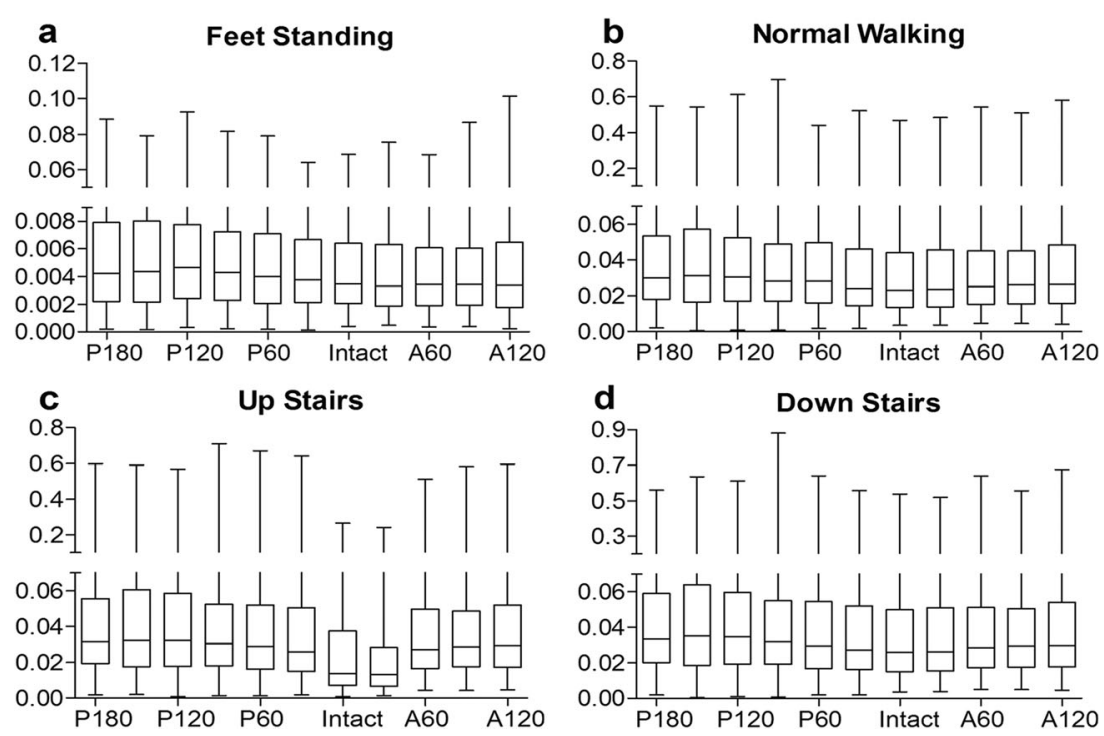

\subsection{Stress and ALLSE}

We analyzed the ALLSE and von Mises stress under four load cases from all the elements on the necrotic region. And then the percentage of von Mises stress and ALLSE remained on the necrotic area after TRO were further calculated, as
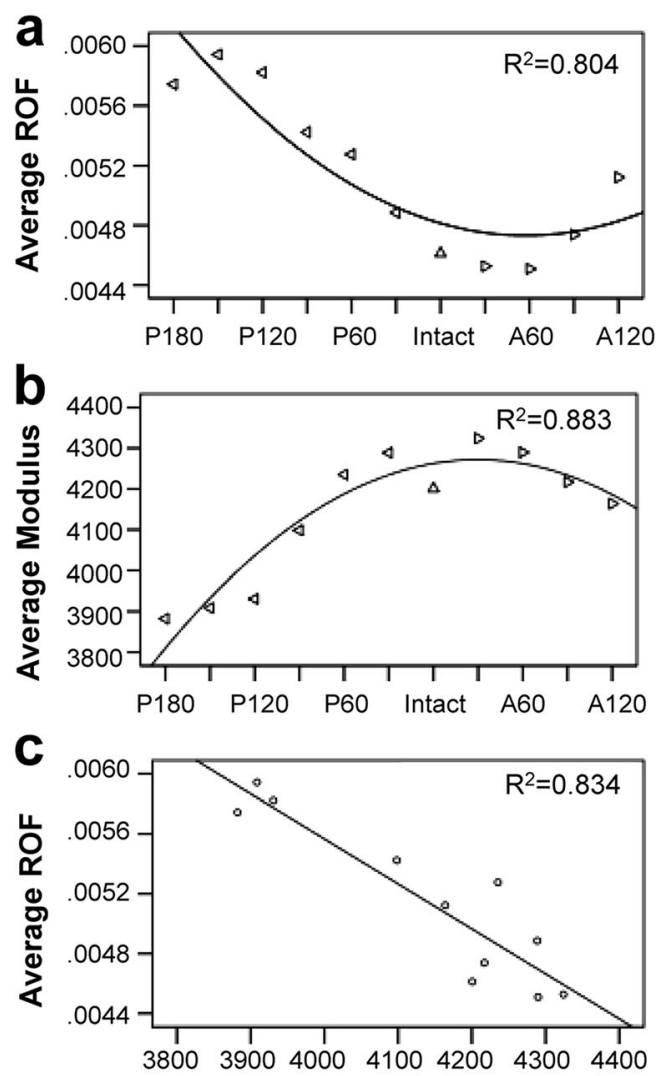

Average Modulus

Fig. 4 The average ROF (a) and modulus (b) of different TRO models. Correlation analysis result (c) presented in Fig. 5a and b. TRO decreased the average von Mises stress and ALLSE effectively by either anterior or posterior rotation. However, the stress and strain energy arise when we rotate posteriorly less than $120^{\circ}$. It demonstrated that the stress reduction by the anterior rotation osteotomy was more effective as compared with that by the posterior rotational osteotomy. Finally, as shown in Fig. 5c, we made a comprehensive evaluation from the three aspects of necrosis expansion trend (ALLSE), necrotic blood supply pressure (stress), and the risk of fracture (ROF). It was observed that $90^{\circ}$ anterior rotational could reach better results, which could comprehensively reduce the risks to $64 \%$.

\subsection{Mechanical experiment validation}

The biomechanical experimental validation results are presented in Fig. 6. The axial stiffness of mechanical experiment and FEA group are $1.7611 \pm 0.1991 \mathrm{~N} / \mu \mathrm{m}$ and $1.9187 \pm$ $0.0547 \mathrm{~N} / \mu \mathrm{m}$, respectively. The corresponding force and displacement data are presented in Fig. 6b. It shows a strong correlation $\left(R^{2}=0.967\right)$. And the strains of the observation areas of DIC and FEA group are $0.3256 \% \pm 0.0567 \%$ and $0.2992 \% \pm 0.0812 \%$, respectively. And the mutation rate is $8.12 \%$.

\section{Discussion}

Osteonecrosis of the femoral head is a disabling disease involving the hips of young patients. Despite many efforts, the etiology and pathogenesis of osteonecrosis still have not been identified. Therefore, treatment methods are various and are often chosen according to stage, location, and size of the necrotic area. Surgery usually involves head preserving procedures or total hip arthroplasty (THA). However, high rates of 


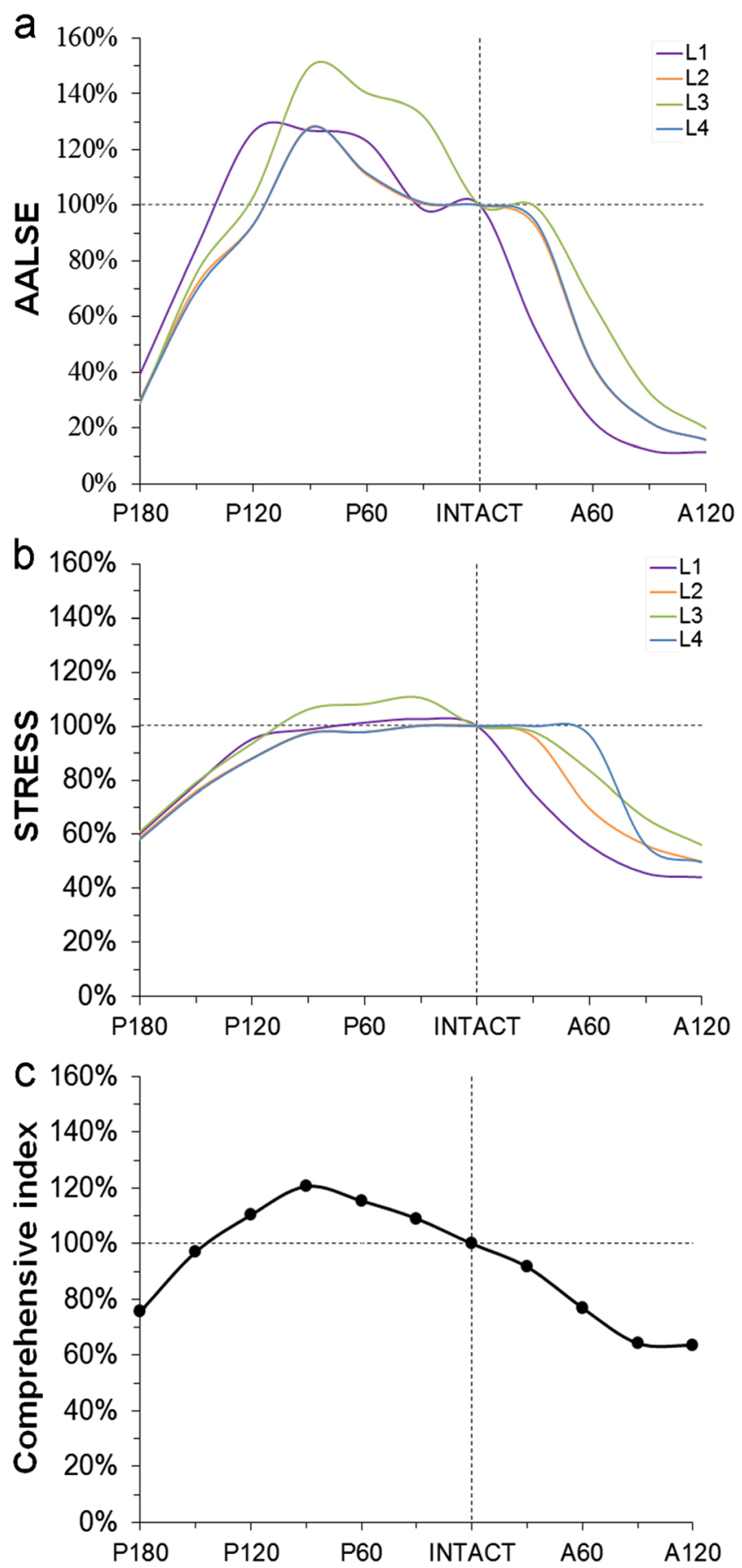

Fig. 5 The percentage of ALLSE (a) and von Mises stress (b) remained on the necrotic area after TRO. c Considering four daily activities and three index values (ALLSE, stress, and ROF) comprehensively

failure have been reported when performing THA in young patients, despite continuous improvement in the design and technique [3, 4]. Besides, the patients are relatively young and with high functional demands, and joint-preserving procedures should be prioritized as much as possible. As a head preserving procedure, TRO procedure itself has been widely used for the treatment of osteonecrosis of the femoral head. It provides a wide exposure and enables rotation of the healthy region to the weight-bearing area, while minimizing the risk of avascular necrosis [6, 26].

Controversy has also swirled around TRO. Fracture nonunion and subsequent collapse are the most discussed. The former is mainly related to the destruction of blood supply, while the latter is mainly related to the postoperative reconstruction of the structural mechanical environment. With a new understanding of the blood supply to the femoral head, we could perform many kinds of improved operations without destruction of blood supply [27]. In terms of mechanical reconstruction, there is lack of literature. Fewer researchers analyzed the stress distribution by FEA method and found the risk of collapse increases as the necrotic area increases. Anterior rotation and a minimum postoperative intact ratio of $34 \%$ were required to achieve successful clinical results [28].

In this study, the most common types of necrosis were analyzed by the FEA method, under multiple operating conditions (four routine activities). The convergence analysis and experiment validation were carried out for ensuring the validity of the model. In this study, two methods were used for validation. As the data of osteonecrosis model is from a living patient, it cannot be verified by direct biomechanical experiment. Therefore, we compared the stress value and distribution with related researches [11, 29], and the similar results were obtained. Meanwhile, the biomechanical experiments were promoted to verify the models from the side. The average axial stiffness of FEA model is $8.95 \%$ greater than our mechanical experiments, while $3.16 \%$ greater than $1.86 \pm$ $0.14 \mathrm{~N} / \mu \mathrm{m}$ of previous reports $[30,31]$. Also the DIC results are very approximate. The above results indirectly prove the validity of our modeling method.

Based on effective finite element models, we made a comprehensive evaluation from the three aspects of necrosis expansion trend (ALLSE), necrotic blood supply pressure (stress), and the risk of fracture (ROF). The results show that the anterior rotational osteotomy is more efficient. Due to vascular limitation, anterior rotational osteotomy should be less than $120^{\circ}$. Thus, we recommend to rotate forward $60^{\circ}$ to $90^{\circ}$ or backward $180^{\circ}$ by considering the anatomy characters, four daily activities and three index values comprehensively. This is consistent with the results of previous studies $[11,12]$. Moreover, we found a strong negative correlation between ROF and bone quality of the roof. Average modulus of the roof area might be an effective indicator of postoperative collapse risk and help us to determine which way to rotate. Besides, we constructed finite element models by gray-scale assignment method. That is convenient for us to observe the internal stress conduction and reflect the mechanics mechanism, which is better than the previous homogenous material assignment.

Since TRO is a high technical demanding procedure, precise preoperative planning is absolutely necessary. Our study 
Fig. 6 The biomechanical experimental validation. a The boundary and loading condition of the FEA group (left) and experimental group (right). The corresponding force and displacement data (b). The anterior surfaces of samples were painted with spray-paint for DIC with white background color and a black random speckle pattern (c). And the average strain results under $2100 \mathrm{~N}$ of the observation areas (left side of $\mathbf{d}$ ) are compared in right side of $\mathbf{d}$. $(n=5)$ a

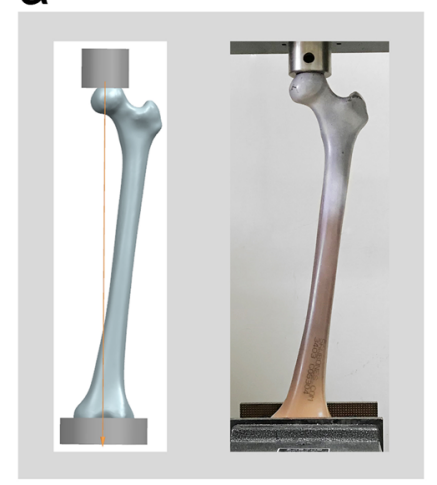

C

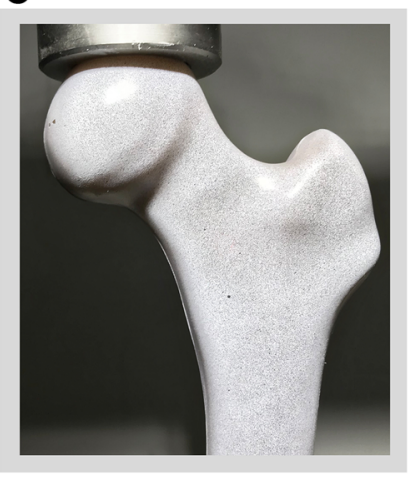

b

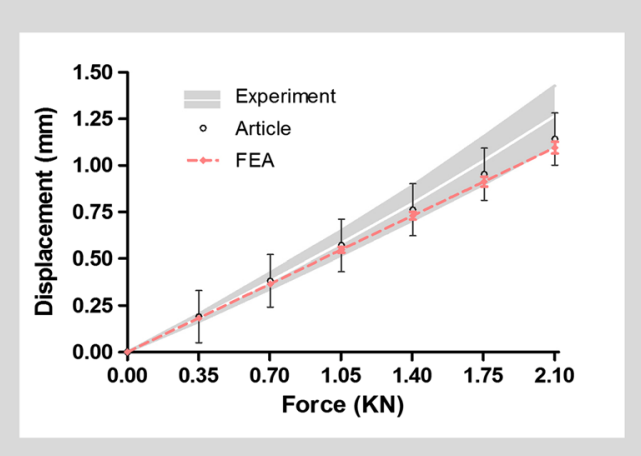

d

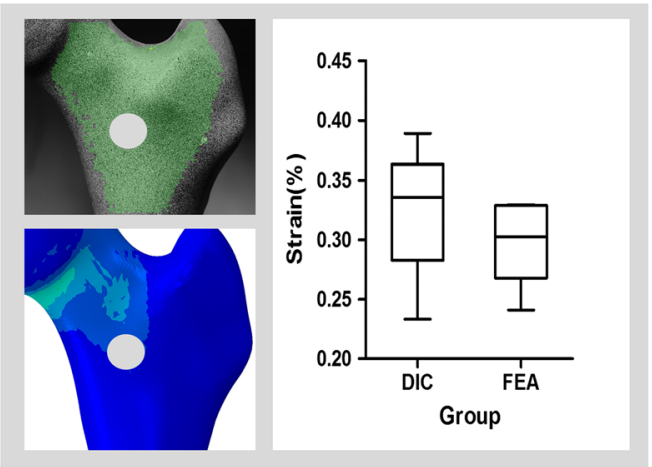

provides a good approach which would be very useful to achieve a good preoperative planning for treatment of patients with femoral head osteonecrosis. However, several limitations should be pointed out. First, since the huge amount of computation, we just analyzed the most common types of osteonecrosis under four routine activities conditions. More types should be considerate in further research. Second, the interfaces on the cutting planes were all tied without taking into account the loosening of the fixation device. Therefore, the results from the FEA might only be interpreted under the well-fixed condition. Third, the femoral FEA models were simplified as isotropy. von Mises criterion does not take into account hydrostatic pressure, and there are some limitations in judging the yield of bone. In the follow-up study, through further experimental research, it is expected to obtain more real and accurate material strength parameters of human bone. Subsequent research of yield criterion and nonlinear analysis will help to further reveal the mechanical mechanism of necrosis and collapse of femoral head.

\section{Conclusions}

In conclusion, TRO technique can effectively reconstruct the mechanical environment of the weight-bearing area of the femoral head, reduce the stress and energy, and collapse the risk of the necrotic area. For the most necrosis located area, anterior and superior position of the femoral head, we suggest to rotate forward $60^{\circ}$ to $90^{\circ}$ or backward $180^{\circ}$ by considering the anatomy characters, four daily activities and three index values comprehensively. Moreover, we firmly believe that digital medicine technology and FEA method can provide more accurate and personalized preoperative planning for patients, which could improve postoperative effect and reduce complications.

Funding information This work was funded by the National Key R\&D Program of China (2017YFC1103400), the Science and Technology Project of Guangdong Province (2016B090917001, 2016B090925001, 2016B090913004, and 2017B090912006), the Sanming Project of Medicine in Shenzhen (SZSM201612019), and the Science Research Program of Foshan City (2017AG100243).

\section{Compliance with ethical standards}

Conflict of interest The authors declare that they have no conflict of interest.

Ethical approval This article does not contain any studies with human participants or animals performed by any of the authors.

Open Access This article is licensed under a Creative Commons Attribution 4.0 International License, which permits use, sharing, adaptation, distribution and reproduction in any medium or format, as long as you give appropriate credit to the original author(s) and the source, provide a link to the Creative Commons licence, and indicate if changes were made. The images or other third party material in this article are included in the article's Creative Commons licence, unless indicated otherwise in a credit line to the material. If material is not included in the article's 
Creative Commons licence and your intended use is not permitted by statutory regulation or exceeds the permitted use, you will need to obtain permission directly from the copyright holder. To view a copy of this licence, visit http://creativecommons.org/licenses/by/4.0/.

\section{References}

1. Mont MA, Cherian JJ, Sierra RJ, Jones LC, Lieberman JR (2015) Nontraumatic osteonecrosis of the femoral head: where do we stand today? A ten-year update. J Bone Joint Surg Am 97:1604-1627

2. Koo KH, Song HR, Yang JW, Yang P, Kim JR, Kim YM (2001) Trochanteric rotational osteotomy for osteonecrosis of the femoral head. J Bone Joint Surg Br 83:83-89

3. Coventry MB, Beckenbaugh RD, Nolan DR, Ilstrup DM (1974) 2 , 012 total hip arthroplasties. A study of postoperative course and early complications. J Bone Joint Surg Am 56:273-284

4. Bessette BJ, Fassier F, Tanzer M, Brooks CE (2003) Total hip arthroplasty in patients younger than 21 years: a minimum, 10year follow-up. Can J Surg 46:257-262

5. Sonoda K, Yamamoto T, Motomura G, Nakashima Y, Yamaguchi R, Iwamoto Y (2015) Outcome of Transtrochanteric rotational osteotomy for posttraumatic osteonecrosis of the femoral head with a mean follow-up of 12.3 years. Arch Orthop Trauma Surg 135: 1257-1263

6. Osawa Y, Seki T, Morita D, Takegami Y, Okura T, Ishiguro N (2017) Total hip Arthroplasty after transtrochanteric rotational osteotomy for osteonecrosis of the femoral head: a mean 10-year follow-up. J Arthroplast 32:3088-3092

7. Nakashima Y, Yamamoto T, Fukushi JI, Motomura G, Hamai S, Kohno Y, Iwamoto Y (2016) Transtrochanteric rotational osteotomy for avascular necrosis of the femoral head after unstable slipped capital femoral epiphysis: 10-year clinical results. J Orthop Sci 21:831-835

8. Okura T, Hasegawa Y, Morita D, Osawa Y, Ishiguro N (2016) What factors predict the failure of curved intertrochanteric varus osteotomy for the osteonecrosis of the femoral head? Arch Orthop Trauma Surg 136:1647-1655

9. Lakhotia D, Swaminathan S, Shon WY, Oh JK, Moon JG, Dwivedi C et al (2017) Healing process of osteonecrotic lesions of the femoral head following transtrochanteric rotational osteotomy: a computed tomography-based study. Clin Orthop Surg 9:29-36

10. Kubo Y, Motomura G, Ikemura S, Sonoda K, Yamamoto T, Nakashima Y (2017) Factors influencing progressive collapse of the transposed necrotic lesion after transtrochanteric anterior rotational osteotomy for osteonecrosis of the femoral head. Orthop Traumatol Surg Res 103:217-222

11. Lee MS, Tai CL, Senan V, Shih CH, Lo SW, Chen WP (2006) The effect of necrotic lesion size and rotational degree on the stress reduction in transtrochanteric rotational osteotomy for femoral head osteonecrosis-a three-dimensional finite-element simulation. Clin Biomech (Bristol, Avon) 21:969-976

12. Chen W, Tai C, Tan C, Shih C, Hou S, Lee MS (2005) The degrees to which transtrochanteric rotational osteotomy moves the region of osteonecrotic femoral head out of the weight-bearing area as evaluated by computer simulation. Clin Biomech 20:63-69

13. Koo KH, Kim R (1995) Quantifying the extent of osteonecrosis of the femoral head. A new method using MRI. J Bone Joint Surg Br 77:875-880

14. Taddei F, Pancanti A, Viceconti M (2004) An improved method for the automatic mapping of computed tomography numbers onto finite element models. Med Eng Phys 26:61-69
15. Bergmann G, Deuretzbacher G, Heller M et al (2002) Hip contact forces and gait patterns from routine activities. J Biomech 35:719 720

16. Langlais F, Fourastier J (1997) Rotation osteotomies for osteonecrosis of the femoral head. Clin Orthop Relat Res:110-123

17. Jones AC, Wilcox RK (2008) Finite element analysis of the spine: towards a framework of verification, validation and sensitivity analysis. Med Eng Phys 30:1287-1304

18. San AT, Ciaccia M, Muller-Karger C, Casanova E (2012) Orientation of orthotropic material properties in a femur FE model: a method based on the principal stresses directions. Med Eng Phys 34:914-919

19. Tran TN, Kowalczyk W, Hohn HP, Jager M, Landgraeber S (2016) Effect of the stiffness of bone substitutes on the biomechanical behaviour of femur for core decompression. Med Eng Phys 38: 911-916

20. Väänänen SP, Amin Yavari S, Weinans H, Zadpoor A, Jurvelin J, Isaksson H (2013) Repeatability of digital image correlation for measurement of surface strains in composite long bones. J Biomech 46:1928-1932

21. Luyckx T, Verstraete M, De Roo K, Van Der Straeten C, Victor J (2016) High strains near femoral insertion site of the superficial medial collateral ligament of the knee can explain the clinical failure pattern. J Orthop Res 34:2016-2024

22. Ali AM, Newman SDS, Hooper PA, Davies CM, Cobb JP (2017) The effect of implant position on bone strain following lateral unicompartmental knee arthroplasty: a biomechanical model using digital image correlation. Bone Joint Res 6:522-529

23. Schileo E, Taddei F, Cristofolini L, Viceconti M (2008) Subjectspecific finite element models implementing a maximum principal strain criterion are able to estimate failure risk and fracture location on human femurs tested in vitro. J Biomech 41:356-367

24. Bayraktar HH, Morgan EF, Niebur GL, Morris GE, Wong EK, Keaveny TM (2004) Comparison of the elastic and yield properties of human femoral trabecular and cortical bone tissue. J Biomech 37 : $27-35$

25. Cilla M, Checa S, Duda GN (2017) Strain shielding inspired redesign of proximal femoral stems for total hip arthroplasty. J Orthop Res 35:2534-2544

26. Shoji T, Yasunaga Y, Yamasaki T, Nakamae A, Mori R, Hamanishi M, Ochi M (2015) Transtrochanteric rotational osteotomy combined with intra-articular procedures for pigmented villonodular synovitis of the hip. J Orthop Sci 20:943-950

27. Park KS, Tumin M, Peni I, Yoon TR (2014) Conversion total hip arthroplasty after previous transtrochanteric rotational osteotomy for osteonecrosis of the femoral head. J Arthroplast 29:813-816

28. Miyanishi K, Noguchi Y, Yamamoto T, Irisa T, Suenaga E, Jingushi $S$ et al (2000) Prediction of the outcome of transtrochanteric rotational osteotomy for osteonecrosis of the femoral head. J Bone Joint Surg Br 82:512-516

29. Bae JY, Kwak DS, Park KS, Jeon I (2013) Finite element analysis of the multiple drilling technique for early osteonecrosis of the femoral head. Ann Biomed Eng 41:2528-2537

30. Gardner MP, Chong AC, Pollock AG, Wooley PH (2010) Mechanical evaluation of large-size fourth-generation composite femur and tibia models. Ann Biomed Eng 38:613-620

31. Heiner AD, Brown TD (2001) Structural properties of a new design of composite replicate femurs and tibias. J Biomech 34:773-781

Publisher's note Springer Nature remains neutral with regard to jurisdictional claims in published maps and institutional affiliations. 


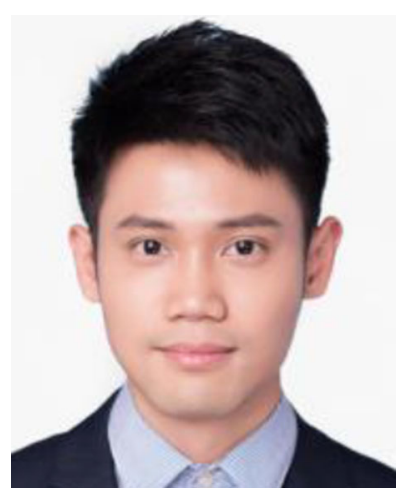

Pusheng Xie is a Ph.D. candidate at the Department of Anatomy of Southern Medical University. His research interests include clinical anatomy, biomedical engineering, digital orthopedics, structure analysis, and topology optimization.

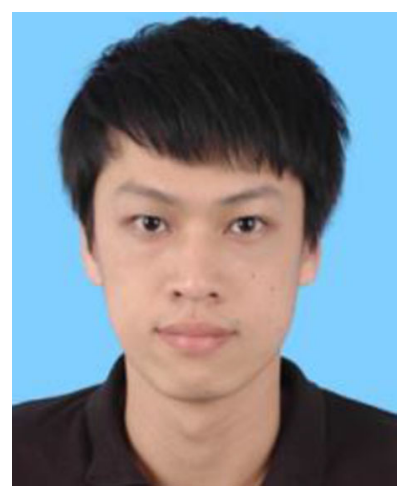

Yuping Deng is a Ph.D. candidate at the Department of Anatomy of Southern Medical University. His research interests include medical threedimensional printing, digital medicine, and finite element methodology.

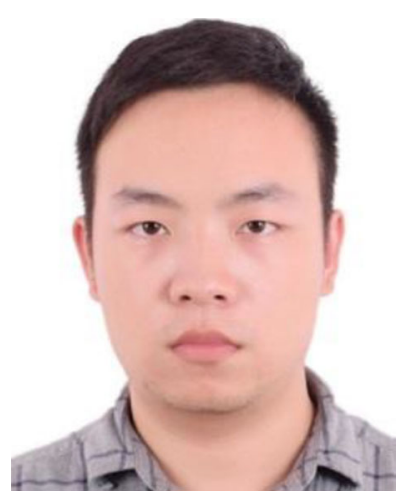

Jinchuan Tan is a Ph.D. candidate at the Department of Anatomy of Southern Medical University. His research interests include clinical anatomy, biomechanics, medical threedimensional printing, and digital medicine.

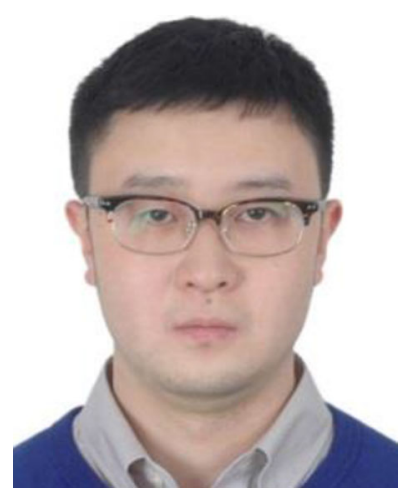

Mian Wang is a Ph.D. candidate at the Department of Anatomy of Southern Medical University. His research interests include medical three-dimensional printing, FEA, and basic science of orthopedic traumatology.

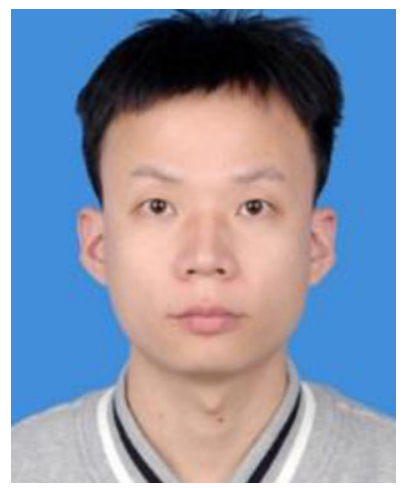

Yang Yang is a Ph.D. candidate at Department of Anatomy, Southern Medical University. His research focuses on medical device design, basic science of orthopedic traumatology, and three-dimensional modeling of human anatomy.

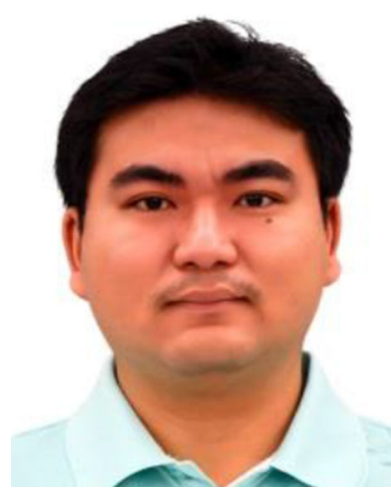

Hanbin Ouyang is a postdoctoral fellow. He specialized in finite element method, biomechanical analysis, and orthopedics. Currently, he is working on a project about the topology optimization for customized orthopedic implant. 


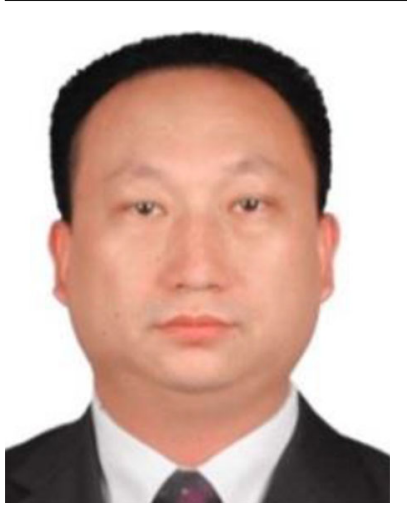

Wenhua Huang works as the Vice-Chairman of Digital Orthopedics Society, Ministry of China of SICOT. His research focuses on clinical anatomy, biomechanics, medical 3D printing, basic science of orthopedic traumatology, etc. 